\title{
Tratamento de água para abastecimento público por ULTRAFILTRAÇÃO: AVALIAÇÃO COMPARATIVA ATRAVÉS DOS CUSTOS DIRETOS DE IMPLANTAÇÃO E OPERAÇÃO COM OS SISTEMAS CONVENCIONAL E CONVENCIONAL COM CARVÃO ATIVADO
}

\author{
DRINKING WATER TREATMENT BY ULTRAFILTRATION: COMPARATIVE EVALUATION \\ THROUGH DIRECT CAPITAL AND OPERATIONAL COSTS WITH CONVENTIONAL AND \\ CONVENTIONAL WITH ACTIVATED CARBON SYSTEMS
}

\section{José CARLOS MIERZWA}

Professor Pesquisador do Departamento de Engenharia Hidráulica e Sanitária Universidade de São Paulo - EPUSP. Coordenador de Projetos do Centro Internacional de Referência em Reúso de Água - CIRRA

\section{MaURício Costa Cabral da SiLVA}

Engenheiro Civil pela Universidade Anhembi Morumbi. São Paulo/SP. Assessor Técnico do Centro Internacional de Referência em Reúso de Água-CIRRA

\section{LUANA Di BEO RODRIGUES}

Engenheira de Produção pela Faculdade de Engenharia Industrial - FEI de São Bernardo do Campo. Assessora Técnica do Centro Internacional de Referência em Reúso de Água-CIRRA

\section{IVANILDO HESPANHOL}

Professor Titular do Departamento de Engenharia Hidráulica e Sanitária da Escola Politécnica da Universidade de São Paulo - EPUSP. Diretor do Centro Internacional de Referência em Reúso de Água - CIRRA

\section{Recebido: 03/04/07 Aceito: 15/02/08}

\section{RESUMO}

Neste trabalho é apresentada uma avaliação comparativa preliminar entre os sistemas de tratamento de água por ultrafiltração, convencional e convencional com carvão ativado, com base nos custos diretos de implantação e operação, utilizando-se os resultados obtidos em uma unidade piloto de ultrafiltração, instalada junto à captação de água no Reservatório Guarapiranga. Para comparação considerou-se os dados relativos à eficiência da remoção de Carbono Orgânico Total. A avaliação de custos foi feita pelo método de cálculo do período de retorno de investimento, obtendo-se valores de $\mathrm{R} \$ 0,20 / \mathrm{m}^{3}$ para o sistema convencional e $\mathrm{R} \$ 0,40 / \mathrm{m}^{3}$ para os outros sistemas. Estes resultados mostram o potencial de processos de separação por membranas no tratamento de água para abastecimento público.

PALAVRAS-CHAVE: Água, abastecimento público; ultrafiltração, custos, PROSAB.

\begin{abstract}
In this work it is presented a comparative evaluation for ultrafiltration, conventional, and conventional with activated carbon water treatment systems, considering direct capital and operational costs, based on the results of an ultrafiltration pilot unit, installed at the Guarapiranga's Reservoir. In order to compare the costs it was considered Total Organic Carbon removal efficiency. Cost evaluation was performed based on the pay back method, resulting in a value of $R \$ 0.20 / \mathrm{m} 3$ for conventional water treatment system, and $R \$ 0.40 / \mathrm{m} 3$ for the others. These results clearly demonstrate the high potential of membrane separation technology for drinking water treatment.
\end{abstract}

KEYWORDS: Water, public supply, ultrafiltration, costs, PROSAB.

\section{INTRODUÇÃO}

O objetivo deste trabalho é apresentar uma avaliação comparativa dos custos diretos de implantação e operação entre os processos de ultrafiltração, convencional e convencional com carvão ativado utilizados no tratamento de água para abasteci- mento público, com base nos resultados de desempenho de uma unidade piloto de ultrafiltração, dados de literatura e consulta a fornecedores de equipamentos. 


\section{REVISÃO DA LITERATURA}

Em decorrência do elevado nível de urbanização, o tratamento de água para abastecimento público passa a ser um desafio, seja pelos grandes volumes de água envolvidos, restrições de área para instalação dos sistemas de tratamento tradicionalmente utilizados e também devido à degradação da qualidade da água dos mananciais disponíveis. Estas condições têm conduzido à busca de alternativas que possibilitem garantir a qualidade da água produzida para abastecimento público. Uma opção que tem sido considerada para atender o desafio da produção de água segura para abastecimento são os processos de separação por membranas, com um significativo avanço nas pesquisas sobre o seu desempenho para aplicação comercial nos últimos anos (Jacangelo, Trussell, Watson, 1997), (Hofman et al, 1998), (Ma et al, 1998), (Wilbert et al, 1998), (Nicolaisen, 2002) e (Bentama et al, 2004).

Dentre os processos de separação por membranas para tratamento de água destacam-se a microfiltração, ultrafiltração, a nanofiltração e a osmose reversa, que utilizam pressão hidráulica como força motriz para separar a água dos contaminantes. A Figura 1 apresenta, esquematicamente, as características dos processos de separação por membranas (Mierzwa, Hespanhol, 2005).

Pela Figura 1 observa-se que os processos de osmose reversa e nanofiltração são os mais eficientes em relação à remoção de contaminantes, porém são os que consomem maior energia, devido à pressão de operação.

No tratamento de água para abastecimento, onde a preocupação são os contaminantes orgânicos, o processo de ultrafiltração pode ser o mais adequado, face o menor consumo de energia e por apresentar eficiência para remover poluentes orgânicos e matéria orgânica natural em função do peso molecular de corte (PMC), da membrana selecionada (Yoon et al, 2006).

No trabalho de Yoon e colaboradores (2006), foram avaliados os processos de nanofiltração $(\mathrm{PMC}=800 \mathrm{~g} / \mathrm{mol})$ e ultrafiltração (PMC $=8.000 \mathrm{~g} / \mathrm{mol})$, para a remoção de 52 compostos orgânicos, incluindo produtos farmacêuticos, de higiene pessoal e desreguladores endócrinos, que foram divididos em dois grupos.
Para os compostos do grupo I (27 compostos), a eficiência de remoção variou de $44 \%$ a $93 \%$ para a nanofiltração e para a ultrafiltração não ultrapassou $40 \%$. Com relação aos compostos do grupo II (25 compostos), a eficiência de remoção foi bastante similar para os dois tipos de membranas, sendo que, para muitos deles, a concentração no permeado estava abaixo do limite de detecção do método.

Gijsbertsen-Abrahamse e colaboradores (2005) estudaram a remoção de cianobactérias e cianotoxinas, também, pelos processos de nanofiltração e ultrafiltração, obtendo resultados mais favoráveis para a remoção de cianotoxinas pelo processo de nanofiltração, embora não tenham sido apresentados os dados relativos às características das membranas utilizadas.

No caso da remoção de matéria orgânica natural, Domany et al (2002), estudaram o desempenho de sistemas de ultrafiltração, utilizando como variáveis de controle a concentração de carbono orgânico total (COT), carbono orgânico dissolvido (COD) e absorção de radiação ultravioleta (UVabs). As membranas utilizadas apresentavam PMC de $5.000 \mathrm{~g} / \mathrm{mol}, 6.000 \mathrm{~g} / \mathrm{mol}$, $15.000 \mathrm{~g} / \mathrm{mol}$ e $100.000 \mathrm{~g} / \mathrm{mol}$. Foram utilizadas soluções sintéticas e amostras de água natural para a realização dos ensaios, obtendo-se remoções próximas a $90 \%$ e $60 \%$, respectivamente, para os quatro tipos de membranas utilizadas.

Para efeito de comparação, o estudo de Chen e colaboradores (2007), apresenta os resultados de ensaios em escala piloto para a remoção de matéria orgânica por sete tipos de processos de tratamento de água, incluindo variações do sistema convencional e convencional avançado, com a utilização de préoxidação com ozônio e permanganato de potássio, carvão ativado granular, cloração e carvão biologicamente ativo. As melhores eficiências de remoção foram obtidas para os sistemas que combinaram o processo convencional de tratamento com o de carvão ativado e pré-oxidação com ozônio, com eficiências próximas de $42 \%$ para a remoção de COT e 61\% para UVabs.

\section{JUSTIFICATIVA}

No Brasil, ao longo das últimas décadas também tem sido observado um intenso processo de urbanização fazendo com que áreas sensíveis, próximas a mananciais para abastecimento sejam ocupados de maneira desordenada, com conseqüente comprometimento da qualidade da água

Um exemplo típico da condição mencionada é a ocupação do entorno do Reservatório Guarapiranga, responsável por cerca de $20 \%$ do abastecimento de água da Região Metropolitana de São Paulo (CETESB, 2003). Tal condição tem exigido um esforço conjunto dos órgãos públicos e da companhia de abastecimento para interromper o processo de degradação ambiental na área do reservatório e, consequentemente, da água (Baltrusis e Ancona, 2006).

Pesquisas sobre os processos de separação por membranas e, conse-

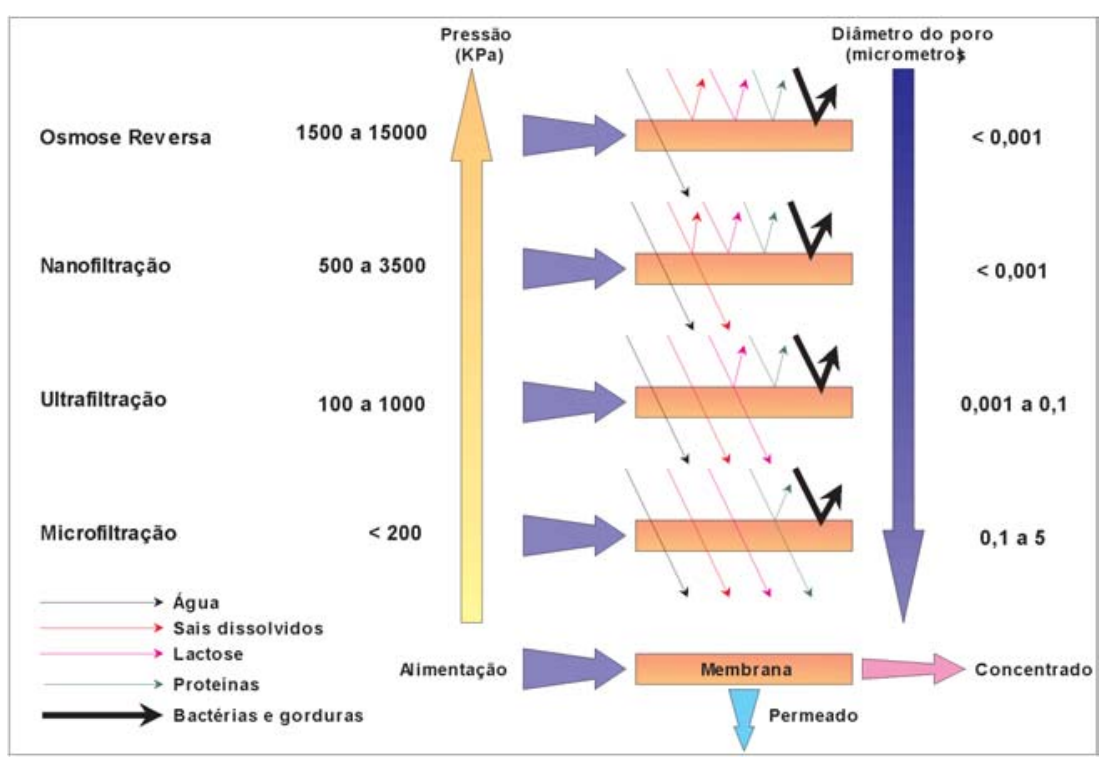

Figura I - Características dos processos de separação por membranas 
qüentemente, sobre a aplicação dessa tecnologia no tratamento de água para abastecimento público são limitadas, mesmo considerando os problemas relacionados à qualidade da água dos mananciais e o avanço tecnológico ocorrido nas últimas décadas, com especial destaque à tecnologia de separação por membranas.

Uma das alegações para que os sistemas de separação por membranas não sejam considerados no tratamento de água para abastecimento público é o seu elevado custo, sem que, no entanto, isto seja devidamente comprovado. Uma das possíveis causas para que se tenha esta percepção é o fato do Brasil não produzir membranas, além do número limitado de empresas que atuam no mercado, mesmo assim com um foco para aplicações industriais, em geral, de pequeno porte (Mierzwa, 2006). Tal condição tem influência direta nos critérios de formação de preços dos sistemas de separação por membranas, comprometendo uma avaliação mais criteriosa da viabilidade econômica da utilização de processos baseados na tecnologia de membranas para tratamento de água de abastecimento.

Assim, o desenvolvimento de uma avaliação comparativa dos custos diretos de implantação e operação entre o processo de ultrafiltração e os processos convencional e convencional com carvão ativado é de grande relevância uma vez que possibilitará demonstrar de maneira mais fundamentada o potencial de aplicação dos sistemas de separação por membranas e também estimular o desenvolvimento de novas linhas de pesquisa no país.

\section{METODOLOGIA}

A avaliação comparativa de custos foi desenvolvida com base nos resultados obtidos em um projeto de pesquisa desenvolvido no âmbito do Programa de Pesquisa em Saneamento Básico - PRO$\mathrm{SAB}$, edital $\mathrm{n}^{\circ} 4$, cujo financiamento foi da Financiadora de Estudos e Projetos - FINEP, para tratamento direto de água de abastecimento por processo de ultrafiltração, utilizando-se membranas enroladas em espiral (Mierzwa, 2006).

Para o desenvolvimento do projeto foi instalada uma unidade piloto próxima ao ponto de captação de água da Companhia de Saneamento Básico do Estado de São Paulo - SABESP, no Reservatório Guarapiranga.
A configuração selecionada para a membrana foi enrolada em espiral da empresa Osmonics, a qual foi adquirida da empresa GE-Water, cujas principais características são apresentadas a seguir (GE Water, 2006).

- Modelo: GK-4040F

- Aplicação: Tratamento de água para remoção de cor e COT

- Peso Molecular de Corte: 3.500 g.mol ${ }^{-1}$

- Material: Filme fino composto (polissulfona e poliamida)

- $\mathrm{pH}$ de operação:

Tratamento - 2 a 11

Limpeza - 2 a 11,5

- Tolerância ao cloro: $500 \mathrm{mg}$ por dia

- Temperatura de operação: até $50{ }^{\circ} \mathrm{C}$

- Área de membrana: 8,36 $\mathrm{m}^{2}$

- Taxa de fluxo: $28,26 \mathrm{~L} \cdot \mathrm{h}^{-1} \cdot \mathrm{m}^{-2} \pm$ $25 \%$ (517 KPa e $25^{\circ} \mathrm{C}$, para água limpa)

- Recuperação de água por módulo: $10 \%$

$\mathrm{Na}$ instalação piloto foi utilizada apenas uma membrana, instalada em um vaso de pressão construído em material polimérico reforçado com fibra de vidro. O fluxograma de processo da unidade piloto está representado na Figura 2, com a indicação dos principais componentes.

A água captada na represa passa pelo filtro de areia, modelo 19CFA4-M, da Jacuzzi, e segue para o tanque de alimentação com capacidade para $500 \mathrm{~L}$, de onde é bombeada para o vaso de pressão, passando pelo filtro tipo tela da Techfilter, para retenção de partículas de $100 \mu \mathrm{m}$. Do vaso de pressão saem duas correntes, o permeado que segue para os tanques de limpeza química $(80 \mathrm{~L})$ e de permeado $(500 \mathrm{~L})$, e o concentrado que é recirculado para a sucção da bomba de alimentação. Periodicamente era feita a descarga do concentrado por meio da abertura de uma válvula solenóide controlada por um temporizador. Com a perda da capacidade de produção do permeado a operação do sistema era interrompida para a realização da limpeza química e sanitização da membrana, utilizando-se soluçốes de hidróxido de sódio $(0,01 \mathrm{M})$, detergente comercial $(0,05 \%)$ e ácido peracético $(0,1 \%)$. Para cada tipo de solução de limpeza utilizada era efetuado o enxágüe com água tratada.

O desempenho do sistema foi avaliado utilizando-se os dados de pro- dução de permeado e recuperação de água, por meio da medida das vazões de permeado e de recirculação, volume de concentrado, pressão de operação e perda de carga na membrana. As medidas foram feitas pelos operadores da SABESP, por meio da observação visual dos instrumentos instalados, a cada duas horas. A vazão de permeado foi medida por um medidor tipo turbina da Burkert, com escala de 0 a $1.000 \mathrm{~L} / \mathrm{h}$, a de recirculação por um rotâmetro com escala de 0 a $100 \mathrm{~L} /$ minutos e a de concentrado por meio de recipiente volumétrico de $10 \mathrm{~L}$ e um cronômetro, já que a descarga era intermitente. A pressão na membrana foi medida através de um manômetro com escala de 0 a $1.000 \mathrm{kPa}$ e a perda de carga por um medidor de pressão diferencial da Gulton, com escala de 0 a $200 \mathrm{kPa}$.

A eficiência de tratamento foi avaliada com base nos resultados de análises físicas, químicas e microbiológicas, das variáveis apresentadas na Tabela 1 . As análises foram realizadas no Laboratório do Centro Internacional de Referência em Reúso de Água - CIRRA e no laboratório da SABESP.

Com os resultados de desempenho da unidade piloto foi elaborado o pré-dimensionamento dos sistemas de tratamento que seriam comparados, ultrafiltração, convencional e convencional com carvão ativado. Para o prédimensionamento foi considerada uma capacidade de produção de $100 \mathrm{~L} / \mathrm{s}$ de água tratada, suficiente para atender a demanda de uma população de 43.200 pessoas, com um consumo per capita de $200 \mathrm{~L} / \mathrm{dia}$.

\section{Sistema de ultrafiltração}

O sistema de ultrafiltração é constituído pelo sistema de pré-tratamento, bombas, membranas, vasos de pressão, instrumentação, sistema de limpeza química e tanques de armazenagem.

Os custos associados à unidade pré-dimensionada foram obtidos mediante consulta a empresa Renics Equipamentos Ltda e outras empresas, com base nos resultados obtidos na unidade piloto instalada no Reservatório Guarapiranga.

Nos custos das membranas e vasos de pressão foi considerado o preço FOB (Free on Board), admitindo-se que a importação de equipamentos para sistemas de tratamento de água para abastecimento é isenta de impostos. 




Figura 2 - Diagrama esquemático da unidade piloto de ultrafiltração instalada junto à captação da SABESP no reservatório Guarapiranga

Para a obtenção do custo de construção e montagem foi considerado um percentual de $20 \%$ em relação ao valor de equipamentos e componentes.

No custo de operação foram considerados: consumo de energia, mão de obra, produtos químicos, disposiçãao dos efluentes e reposição de membranas, adotando-se uma vida útil de cinco anos.

\section{Sistema convencional e convencional com carvão ativado}

O sistema convencional considerado foi o de ciclo completo, ou seja, coagulaçãa, floculação, sedimentação, filtração e desinfecção com cloro, operando nas mesmas condições que opera o atual sistema que capta água do reservatório
Guarapiranga em São Paulo/SP. Em razão da problemática associada ao lodo produzido, neste sistema também foi considerada a implantação de uma unidade para condicionamento do lodo.

Os custos para o sistema convencional foram obtidos mediante consulta a fornecedores especializados e também com base em informações obtidas junto a SABESP, principalmente com relação à dosagem de produtos químicos e seus custos, assim como do valor de investimento em instrumentação e controle do sistema.

Para efeito de comparação, foi feito o levantamento do custo de implantação de uma estação de tratamento convencional com base em uma curva de custo, obtida a partir dos dados sobre os custos de alguns sistemas implantados em países em desenvolvimento
(Schulz, Okun, 1984), atualizados pelo Construction Cost Index, da Engineering News Record, para março de 2006.

Em relação ao sistema de carvão ativado, considerando a utilização de leitos de carvão ativado granular com tempo de contato de 15 minutos (Empty Bed Contact Time), o custo foi obtido por meio de consulta telefônica a uma empresa que comercializa equipamentos e sistemas para tratamento de água. Para efeito de comparação, foi verificado o custo de sistemas de tratamento por carvão ativado granular disponibilizado pela Agência Americana de Proteção Ambiental (EPA, 2005).

A obtenção dos custos relativos ao sistema convencional com carvão ativado resultou da soma dos custos do sistema convencional e de carvão ativado. 


\section{Avaliação de custos}

A avaliação comparativa dos custos diretos dos sistemas considerados foi feita com base no período de retorno de investimentos, através da expressão clássica de custos, Equação 1 (Hirschfeld, 1982), utilizando-se como base o valor do metro cúbico de água produzido.

Custo $=\frac{\mathrm{P}\left[\frac{\mathrm{i}(1+\mathrm{i})^{\mathrm{n}}}{(1+\mathrm{i})^{\mathrm{n}}-1}\right]+\mathrm{O}}{\mathrm{V}}$

Onde:

Custo: Valor do metro cúbico de água tratada;

P: Valor do investimento;

O: Gasto anual com a operação do sistema;

$\mathrm{V}$ : Volume anual de água produzido $\left(\mathrm{m}^{3}\right)$;

i:Taxa de retorno do investimento (\% a.a./100);

n: Número de anos para o retorno do investimento.

A partir dos dados sobre custos de investimento e de operação para os sistemas de tratamento considerados, adotando-se uma taxa de retorno do investimento, referente à Ata de reuniāo do COPOM de 31/05/2006, de 15,25 \% a.a. (http://www.bcb.gov. br/?COPOMJUROS), foram obtidos os custos para tratamento, por meio de uma planilha em Excel $^{\circledR}$.

Sempre que necessário, a conversão de valores foi feita considerando-se a taxa de câmbio de 02 de junho de 2006, R\$2,29/US\$.

\section{RESULTADOS E DISCUSSÕES}

A unidade piloto foi mantida em operação contínua de agosto de 2005 a agosto de 2006, totalizando mais de 7.800 horas de operação. Os dados de vazão normalizada de permeado a $25^{\circ} \mathrm{C}$, obtidas durante a operação da unidade piloto são apresentados na Figura 3 e os parâmetros médios de operação na Tabela 2.

A melhoria no desempenho do sistema de ultrafiltração observada a partir de janeiro de 2006 foi devida a introdução de uma etapa de pré-cloração da água bruta, antes da passagem pelo filtro de areia, utilizando-se uma dosagem suficiente para atender a demanda de cloro, aproximadamente $0,2 \mathrm{mg} / \mathrm{L}$. As operaçóes de limpeza química foram realizadas entre cada período de operação apresentado no eixo das abscissas do gráfico da Figura 3.

Os dados sobre a eficiência de remoção dos contaminantes considerados para a avaliação de desempenho da unidade de ultrafiltração estão apresentados nas Tabelas 3 e 4.

Nas análises para microcistinas, em nenhuma amostra de água bruta ou de permeado foi detectada a sua presença. Contudo, em quatro amostras do concentrado de 2006, foram obtidas concentraçōes variando de $0,13 \mu \mathrm{g} / \mathrm{L}$ a $0,18 \mu \mathrm{g} / \mathrm{L}$, ligeiramente superiores ao limite de detecção do método, que é de $0,1 \mu \mathrm{g} / \mathrm{L}$. Tanto para o etinilestradiol, quanto para o nonilfenol, em nenhuma das amostras analisadas foi detectada a presença destas substâncias, inclusive no concentrado.

Tabela I - Variáveis de qualidade utilizadas para avaliar o desempenho da unidade piloto de ultrafiltração

\begin{tabular}{|c|c|c|}
\hline Parâmetro & $\begin{array}{c}\text { Analisados no } \\
\text { Laboratório do } \\
\text { CIRRA }^{a}\end{array}$ & $\begin{array}{c}\text { Analisados no } \\
\text { Laboratório } \\
\text { da SABESP }\end{array}$ \\
\hline Absorção de radiação UV a 254 nm & $\mathrm{X}$ & \\
\hline Alcalinidade total & $\mathrm{X}$ & $\mathrm{X}$ \\
\hline Carbono orgânico total & & $\mathrm{X}$ \\
\hline Coliformes totais & & $\mathrm{X}$ \\
\hline Condutividade elétrica & $\mathrm{X}$ & \\
\hline Contagem de algas & & $\mathrm{X}$ \\
\hline Cor aparente & $\mathrm{X}$ & $\mathrm{X}$ \\
\hline Dureza total & $\mathrm{X}$ & \\
\hline Escherichia Coli & & $\mathrm{X}$ \\
\hline Etinilestradiol ${ }^{\mathrm{c}}$ & $\mathrm{X}$ & \\
\hline Ferro & & $X$ \\
\hline Fósforo total & $\mathrm{X}$ & \\
\hline Manganês & & $\mathrm{X}$ \\
\hline Microcistinas $^{c}$ & $\mathrm{X}$ & \\
\hline Nitrogênio Kjeldahl e amoniacal & $\mathrm{X}$ & \\
\hline Nonilfenol c & $\mathrm{X}$ & \\
\hline $\mathrm{pH}$ & $\mathrm{X}$ & $\mathrm{X}$ \\
\hline Turbidez & $\mathrm{X}$ & $\mathrm{X}$ \\
\hline
\end{tabular}

- quando não for especificado utilizou-se os procedimentos estabelecidos no Standards Methods for the Examination of Water and Wastewater. $20^{\text {th }}$ edition.

b - as análises foram realizadas pela SABESP, seguindo-se os mesmos procedimentos utilizados para o controle da qualidade da água de suas estaçóes.

- Análise pelo método de enzima conjugada e imunosorbente (ELISA), utilizando-se Kits da BEACON Analytical Systems Inc e da Abraxis Kits para microcistinas de da Abraxis Kits para os demais contaminantes.

Tabela 2 - Parâmetros médios de operação da unidade piloto no período de agosto de 2005 a agosto de 2006

\begin{tabular}{cc}
\hline Parâmetro operacional & Valor \\
\hline Vazão de permeado $\left(\mathrm{L} / \mathrm{h}\right.$ a $\left.25^{\circ} \mathrm{C}\right)$ & $179,5 \pm 29,53$ \\
Vazão de concentrado $\left(\mathrm{L} / \mathrm{h}\right.$ a $\left.25^{\circ} \mathrm{C}\right)$ & $32,0 \pm 10,43$ \\
Vazão de recirculação $\left(\mathrm{L} / \mathrm{h}\right.$ a $\left.25^{\circ} \mathrm{C}\right)$ & $2.173,5 \pm 391,14$ \\
Pressão de operação $(\mathrm{kPa})$ & $418,3 \pm 34,31$ \\
Taxa de fluxo normalizada $\left(\mathrm{L} / \mathrm{d} \cdot \mathrm{m}^{2} \cdot \mathrm{kPa}\right)$ & $1,3 \pm 0,3$ \\
Recuperação de água $(\%)$ & $85,0 \pm 3,61$ \\
\hline
\end{tabular}




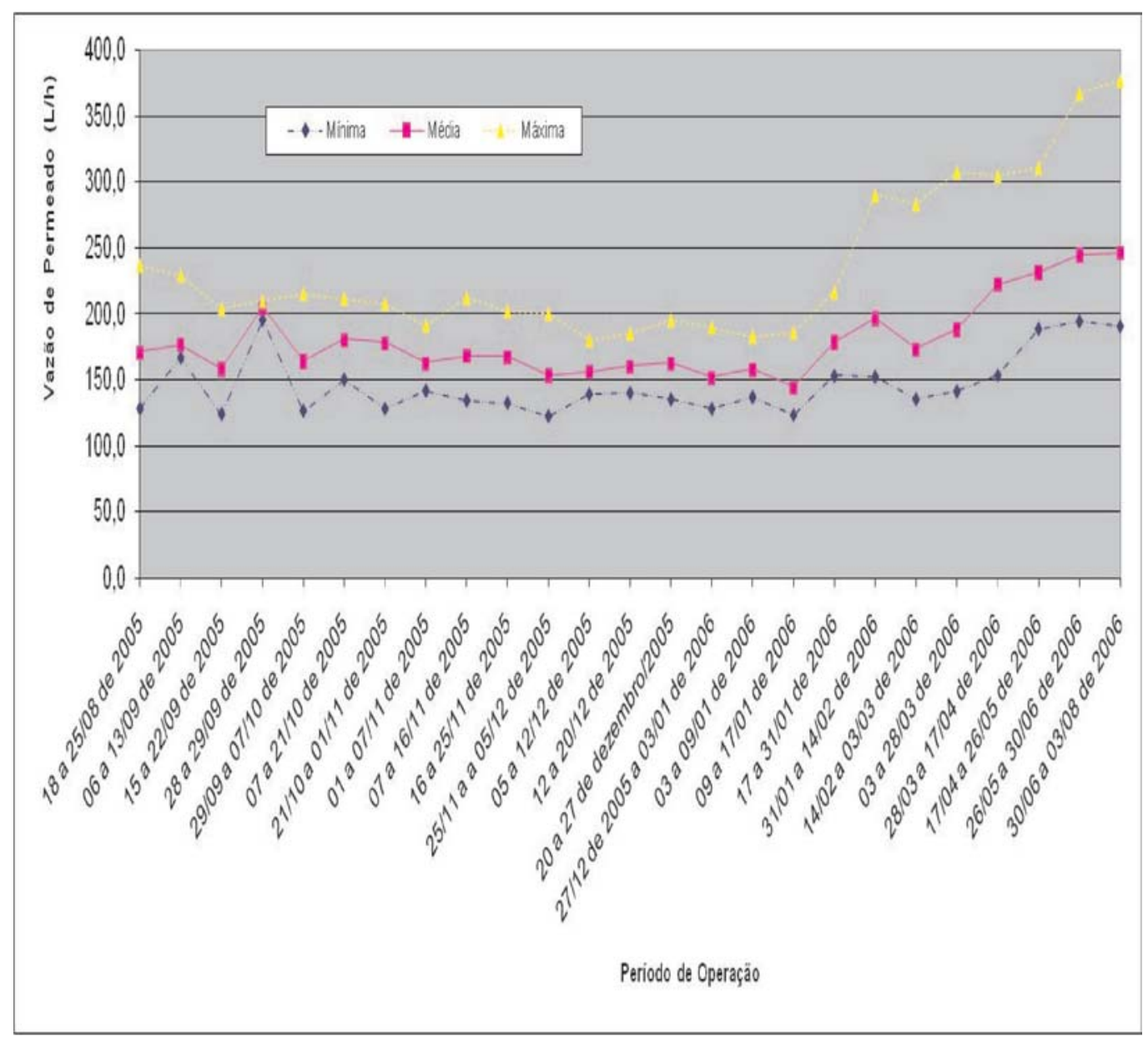

Figura 3 - Vazão normalizada de permeado da unidade de ultrafiltração durante o ensaio com a unidade piloto

Tabela 3 - Eficiência da unidade piloto para remoção de contaminantes a partir das análises realizadas no laboratório do CIRRA

\begin{tabular}{|c|c|c|c|c|c|c|}
\hline \multirow[t]{2}{*}{ Parâmetro } & \multirow{2}{*}{$\begin{array}{c}\text { No de } \\
\text { amostras }\end{array}$} & \multicolumn{2}{|c|}{ Água bruta } & \multicolumn{2}{|c|}{ Permeado } & \multirow{2}{*}{$\begin{array}{l}\text { Eficiência } \\
\text { de remoção } \\
(\%)\end{array}$} \\
\hline & & $\begin{array}{l}\text { Valores } \\
\text { médios }\end{array}$ & $\begin{array}{l}\text { Desvio } \\
\text { padrão }\end{array}$ & $\begin{array}{l}\text { Valores } \\
\text { médios }\end{array}$ & $\begin{array}{l}\text { Desvio } \\
\text { padrão }\end{array}$ & \\
\hline Absorção de Radiação UV $\left(\mathrm{cm}^{-1}\right)$ & 17 & 0,09 & 0.01 & 0,03 & 0,01 & $66,7 \%$ \\
\hline Alcalinidade $\left(\mathrm{mg} \mathrm{CaCO}_{3} / \mathrm{L}\right)$ & 20 & 40,98 & 5,09 & 37,02 & 2,15 & $9,7 \%$ \\
\hline Condutividade elétrica $\left(\mu \mathrm{S} / \mathrm{cm}^{-1}\right)$ & 25 & 141,86 & 16,63 & 133,92 & 14,45 & $5,6 \%$ \\
\hline Cor aparente (mg Pt-Co/L) & 22 & 29,1 & 8,87 & 1,8 & 1,27 & $93,7 \%$ \\
\hline Dureza total $\left(\mathrm{mg} \mathrm{CaCO}_{3} / \mathrm{L}\right)$ & 22 & 33,36 & 1,82 & 29,80 & 2,27 & $10,7 \%$ \\
\hline Fósforo Total (mg P/L) & 11 & 0,03 & 0,01 & 0,02 & 0,01 & $33,3 \%$ \\
\hline Microcistinas $(\mu \mathrm{g} / \mathrm{L})$ & 12 & $<0,1$ & $<0,1$ & $<0,1$ & $--\mathrm{x}--$ & $--\mathrm{x}--$ \\
\hline Nitrogênio Amoniacal (mg N/L) & 10 & 0,17 & 0,1 & 0,19 & 0,16 & $--\mathrm{x}--$ \\
\hline Nitrogênio Kjeldahl (mg N/L) & 12 & 1,83 & 1,14 & 0,83 & 0,67 & $54,6 \%$ \\
\hline Nitrogênio orgânico (mg N/L) & 9 & 1,30 & 0,44 & 0,60 & 0,48 & $53,8 \%$ \\
\hline pH (unidades) & 23 & 7,52 & 0,37 & 7,36 & 0,22 & $--\mathrm{x}--$ \\
\hline Turbidez (uT) & 21 & 3,02 & 0,92 & 0,20 & 0,12 & $93,4 \%$ \\
\hline
\end{tabular}


Tabela 4 - Eficiência da unidade piloto para remoção de contaminantes a partir das análises realizadas no laboratório da SABESP

\begin{tabular}{|c|c|c|c|c|c|c|}
\hline \multirow[t]{2}{*}{ Parâmetro } & \multirow{2}{*}{$\begin{array}{c}\text { No de } \\
\text { amostras }\end{array}$} & \multicolumn{2}{|c|}{ Água Bruta } & \multicolumn{2}{|c|}{ Permeado } & \multirow{2}{*}{$\begin{array}{c}\text { Eficiência } \\
\text { de remoção } \\
(\%)\end{array}$} \\
\hline & & $\begin{array}{l}\text { Valores } \\
\text { médios }\end{array}$ & $\begin{array}{l}\text { Desvio } \\
\text { padrão }\end{array}$ & $\begin{array}{l}\text { Valores } \\
\text { médios }\end{array}$ & $\begin{array}{l}\text { Desvio } \\
\text { padrão }\end{array}$ & \\
\hline Alcalinidade $\left(\mathrm{mg} \mathrm{CaCO}_{3} / \mathrm{L}\right)$ & 79 & 33,20 & 1,8 & 31,46 & 2,07 & $5,24 \%$ \\
\hline Carbono Orgânico Total (mg C/L) & 24 & 4,57 & 0,38 & 1,08 & 0,59 & $76,37 \%$ \\
\hline Coliformes Totais (NMP/100 mL) & 18 & $1,47 \mathrm{E}+04$ & $3,70 \mathrm{E}+04$ & $0,00 \mathrm{E}+00$ & $0,00 \mathrm{E}+00$ & $100,00 \%$ \\
\hline Contagem de Algas (UPA/mL) & 27 & $7,09 \mathrm{E}+03$ & $9,40 \mathrm{E}+03$ & $7,60 \mathrm{E}-01$ & $9,40 \mathrm{E}-01$ & $99,99 \%$ \\
\hline Cor aparente $(\mathrm{mg} \mathrm{Pt}-\mathrm{Co} / \mathrm{L})$ & 80 & 32,6 & 17,8 & 3,7 & 1,25 & $88,79 \%$ \\
\hline Escherichia Coli (NMP/100 mL) & 18 & $2,42 \mathrm{E}+02$ & $4,85 \mathrm{E}+02$ & $0,00 \mathrm{E}+00$ & $0,00 \mathrm{E}+00$ & $100,00 \%$ \\
\hline Ferro $(\mathrm{mg} \mathrm{Fe} / \mathrm{L})$ & 81 & 0,02 & 0,03 & 0,01 & 0,02 & $50,00 \%$ \\
\hline Manganês (mg Mn/L) & 81 & 0,03 & 0,05 & 0,01 & 0,02 & $66,67 \%$ \\
\hline pH (unidades) & 82 & 7,40 & 0,24 & 7,24 & 0,17 & $--\mathrm{x}--$ \\
\hline Turbidez & 82 & 2,89 & 0,87 & 0,13 & 0,07 & $95,50 \%$ \\
\hline
\end{tabular}

Com os dados de desempenho da unidade piloto foi possível desenvolver o pré-dimensionamento de um sistema de tratamento para a realização da avaliação de custos. Ressalta-se que foram considerados apenas os custos relacionados aos principais componentes do sistema.

$\mathrm{Na}$ Tabela 5 são apresentados os custos relativos à implantação do sistema de ultrafiltração com capacidade para $100 \mathrm{~L} / \mathrm{s}$ de água tratada, divido em três módulos e na Tabela 6 os custos para a operação da unidade. Estes custos foram obtidos através de consulta à empresa Renics Equipamentos Ltda, em setembro de 2006.

Para efeito de comparação, foi utilizada a curva de custos de sistemas de ultrafiltração, Equação 2, disponibilizada no manual da EPA (EPA, 2005).

Custo $=341.615^{*}$ Capacidade do sistema +345.942

Onde:

Custo: Custo de investimento (US\$); Capacidade do sistema: Capacidade de produção (milhões de galōes por dia).

Com base na Equação 2 e na capacidade da unidade considerada (2,28 mgd), o custo de investimento do sistema de ultrafiltração é de US\$1.124.824,20, ou R \$2.575.847,42, que resulta em uma diferença inferior a $10 \%$ ao levantamento de custo efetuado, indicando a sua adequação para efeito de uma comparação preliminar.

$\mathrm{Na}$ Tabela 7 são apresentadas as principais unidades e componentes dos
Tabela 5 - Custo de investimento no sistema de tratamento de água por ultrafiltração

\begin{tabular}{cc}
\hline Componente & Custo (R\$) \\
\hline Membranas & $1.134 .000,00$ \\
Vasos de pressão & $227.430,00$ \\
Bombas de alta pressão & $129.000,00$ \\
Pré-filtros de areia & $38.655,00$ \\
Estrutura metálica & $21.000,00$ \\
Instrumentação e controle & $159.000,00$ \\
Sistema de dosagem de cloro & $66.885,00$ \\
Sistema de limpeza química & $160.062,08$ \\
Subtotal & $1.936 .032,08$ \\
Construção e montagem & $387.206,42$ \\
Custo do terreno $\left(250 \mathrm{~m}^{2}\right)$ & $50.000,00$ \\
Custo Total do Sistema & $2.373 .238,50$ \\
\hline
\end{tabular}

Tabela 6 - Custo operacional anual do sistema de tratamento por ultrafiltração

\begin{tabular}{cc}
\hline Item & Custo anual $(\mathrm{R} \$)$ \\
\hline Mão de obra & $93.381,60$ \\
Produtos químicos & $54.234,77$ \\
Reposição de membranas ${ }^{(1)}$ & $226.800,00$ \\
Custo com energia & $463.038,07$ \\
Custo de disposição de efluentes ${ }^{(2)}$ & $60.795,00$ \\
Custo total de operação & $898.249,44$
\end{tabular}

(1) $\mathrm{O}$ custo relacionado à reposição das membranas considera uma vida útil das membranas de cinco anos, de forma que o valor anual é um quinto do custo total das membranas, apresentado na Tabela 4. (2) $\mathrm{O}$ custo relativo à disposição de efluentes refere-se ao volume gerado nas operações de limpeza química, adotando-se uma tarifa para disposição de $\mathrm{R} \$ 8,75 / \mathrm{m}^{3}$. 
sistemas convencional e carvão ativado, com os respectivos custos, obtidos através de consulta a fornecedores, à SABESP e a literatura.

A Figura 4 apresenta a curva de custo de estaçóes de tratamento de água para abastecimento, utilizada para comparação com os custos obtidos no levantamento efetuado mediante consulta a fornecedores e a SABESP.

Pela Figura 4, para um sistema com capacidade para $100 \mathrm{~L} / \mathrm{s}$, verifica-se que o custo de implantação é de US\$ 504.386,89, ou R\$1.150.002,11, próximo ao valor obtido pelo levantamento efetuado, considerando-se a inclusão do sistema para desidratação de lodo no custo resultante da figura.

Também foi verificado o custo de sistemas de tratamento por carvão ativado granular disponibilizado pela Agência Americana de Proteção Ambiental (EPA, 2005). Para um sistema com capacidade equivalente à avaliada foi obtido um valor próximo de 1,5 milhôes de dólares, o que permite utilizar com segurança o valor informado pela empresa consultada, ressaltando-se que no custo apresentado pela EPA está contemplado o valor da regeneração do leito de carvão.

Os custos relativos à operação do sistema foram obtidos considerando-se, principalmente, as despesas com mão de obra, produtos químicos, disposição de lodo e reposição de materiais, conforme mostra a Tabela 8 .

No custo relativo à troca de carvão foram consideradas duas cargas anuais, ressaltando-se que, para o caso de remoção de matéria orgânica natural, este valor é conservativo (Chen; Jeng; Chen, 1996).

Com base na equação 1 e nos dados das Tabelas 5 a 8 , foi obtida a estimativa dos custos de tratamento de água para os sistemas considerados, apresentados na Tabela 9.

Analisando os dados da Tabela 9 verifica-se que o sistema convencional de tratamento de água apresenta um custo de produção significativamente menor que o sistema de ultrafiltração. Contudo, para o caso de mananciais que apresentam problemas de qualidade de água, onde o emprego de carvão ativado é necessário, os custos se equiparam, mostrando o potencial dos sistemas de separação por membranas para tratamento de água para abastecimento público.
Tabela 7 - Custos para o sistema de tratamento de água pelo processo convencional e com unidade complementar de tratamento por carvão ativado granular

\begin{tabular}{cc}
\hline Componente & Custo $(\mathrm{R} \$)$ \\
\hline Calha Parshall & $12.500,00$ \\
Floculador & $84.536,17$
\end{tabular}

Sedimentador de alta taxa

$136.908,79$

Filtros de areia e antacito

$103.011,34$

Câmara de desinfecção

Sistema de dosagem de produtos

$96.400,00$ químicos

Sistema de dosagem de cloro $\quad 66.885,00$

Adensador de lodo

$7.667,18$

Filtro prensa

$150.000,00$

Instrumentação e controle

$200.000,00$

Casa de química

$35.100,00$

Subtotal

Construção e montagem

$284.525,49$

Custo do terreno $\left(1000 \mathrm{~m}^{2}\right)$

$200.000,00$

Custo total do sistema convencional

$1.432 .943,78$

Sistema de carvão ativado

$1.100 .000,00$

Construção e montagem

$220.000,00$

Sistema de carvão ativado

$1.320 .000,00$

Custo total

$2.752 .943,78$



Fonte: Schulz e Okun, 1984

Figura 4 - Curva de custo de estações de tratamento de água para abastecimento 
Tabela 8 - Custo operacional anual do sistema convencional de tratamento de água sem e com a unidade de carvão ativado granular

\begin{tabular}{cc}
\hline Item & Custo anual (R\$) \\
\hline Mãstema sem carvão ativado obra & \\
Produtos químicos & $186.763,20$ \\
Reposição de areia (10 \% a.a) & $97.400,20$ \\
Reposição de antracito (10 \% a.a) & 226,80 \\
Disposição de lodo & $2.520,00$ \\
Custo do sistema convencional & $29.280,00$ \\
Sistema com carvão ativado & $316.190,20$ \\
Custo de energia & $82.918,66$ \\
Troca do Carvão & $344.000,00$ \\
Custo do sistema de carvão ativado & $426.918,66$ \\
Custo total de operação & $743.108,86$ \\
\hline
\end{tabular}

Tabela 9 - Custos de tratamento de água em função do período de retorno de investimento

\begin{tabular}{cccc}
\hline $\begin{array}{c}\text { Período de retorno } \\
(\text { anos })\end{array}$ & $\begin{array}{c}\text { Custo de tratamento }\left(\mathrm{R} \$ / \mathrm{m}^{3}\right) \\
\text { Sistema } \\
\text { convencional }\end{array}$ & $\begin{array}{c}\text { Sistema convencional } \\
\text { com carvão ativado }\end{array}$ & $\begin{array}{c}\text { Sistema de } \\
\text { ultrafiltração }\end{array}$ \\
\hline 1 & 0,63 & 1,24 & 1,15 \\
5 & 0,24 & 0,50 & 0,51 \\
10 & 0,19 & 0,41 & 0,44 \\
15 & 0,18 & 0,39 & 0,42 \\
20 & 0,17 & 0,38 & 0,41 \\
25 & 0,17 & 0,37 & 0,40 \\
30 & 0,17 & 0,37 & 0,40 \\
\hline
\end{tabular}

Ressalta-se também que os custos dos sistemas de separação por membranas apresentam um grande potencial para serem reduzidos em função da ampliação do uso desta tecnologia, ao contrário do que ocorre com os sistemas convencionais, principalmente pela necessidade da complementação do tratamento por outras tecnologias, que apresentam custos elevados e já são bem estabelecidas no mercado.

\section{CONCLUSÕES}

A operação de uma unidade piloto de ultrafiltração permitiu a obtenção de dados para a realização de uma avaliação comparativa de custos diretos de implantação e operação entre os sistemas convencional e convencional com carvão ativado.
Pelos resultados obtidos verifica-se que a membrana de ultrafiltração selecionada apresenta uma boa eficiência para a remoção de compostos orgânicos e matéria orgânica natural, considerando-se os resultados para COT, eficiência superior a 76\%, e Absorção de Radiação Ultravioleta, eficiência superior a $66 \%$.

Com base nos resultados de desempenho da unidade piloto de ultrafiltração foi possível desenvolver o pré-dimensionamento de sistemas de tratamento de água, considerando-se apenas os componentes principais.

Uma avaliação comparativa entre os sistemas de ultrafiltração, convencional e convencional com carvão ativado, baseada em um levantamento de custos com fornecedores, companhia de abastecimento e literatura, demonstra o menor custo de produção associado ao sistema convencional (próximo de $\left.\mathrm{R} \$ 0,20 / \mathrm{m}^{3}\right)$. Contudo, considerando-se a produção de água com o mesmo grau de qualidade, onde é necessário implantar sistemas complementares ao sistema convencional, o sistema de ultrafiltração se mostra bastante competitivo quando da utilização de um sistema de carvão ativado em complementação ao sistema convencional, com um custo de tratamento próximo de $\mathrm{R} \$ 0,40 / \mathrm{m}^{3}$.

Estes resultados, embora baseados em uma comparação de custos diretos, demonstram o potencial de utilização dos processos de separação por membranas para o tratamento de água para abastecimento, ao mesmo tempo em que eliminam algumas distorçôes sobre a percepção de seus custos. Isto cria um panorama favorável para a ampliação de estudos e pesquisas relacionados aos processos de separação por membranas, não apenas para a sua aplicação, mas para uma análise de custos mais detalhada e também para fabricação de membranas e equipamentos.

\section{REFERÊNCIAS}

BALTRUSIS, N.; ANCONA, A.L. Recuperação ambiental e saúde pública. O programa Guarapiranga. Saúde e Sociedade v. 15, n. 1, p. 9-21, jan.-abr. 2006.

BENTAMA, J. et al. Technological innovation for the production of drinking water by membrane processes. Desalination 168, p. 283-286. 2004.

CETESB, São Paulo. Relatório de qualidade das águas interiores do estado de São Paulo 2002/ CETESB. São Paulo: CETESB, 2003.

CHEN, C. et al. Comparison of seven kinds of drinking water treatment processes to enhance organic material removal: A pilot test. Science of the Total Environment 382, p. 93-102. 2007.

CHEN, P. H.; JENG, C.H.; CHEN, K.M. Evaluation of granular activated carbon for removal of trace organic compounds in drinking water. Environment International, Vol. 22, No 3, p. 343-359. 1996.

DOMANY, Z. et al. Humic substances removal from drinking water by membrane filtration. Desalination 145, p. 333-337. 2002.

EPA. Technologies and Costs - Document for the Final Long Term 2 Enhanced Surface Water Treatment Rule and Final Stage 2 Disinfectants and Disinfection Byproducts Rule. EPA 815-R-05-013, December 2005. disponível em http://www.epa. gov/safewater.

HOFMAN, J.A.M.H. et al. Enhanced surface water treatment by ultrafiltration. Desalination 119, p. 113-125. 1998 .

GIJSBERTSEN-ABRAHAMSE, A.J. et al. Removal of cyanotoxins by ultrafiltration and nanofiltration. Journal of Membrane Scicence xxx (2005).p. xxx-xxx. Article in press. 
HIRSCHFELD, H. Engenharia Econômica. 2a edição, Editora Atlas S/A. São Paulo, 334 p. 1982.

JACANGELO, J.G; TRUSSELL, R.R; WATSON, M. Role of membrane technology in drinking water treatment in the United States. Desalination, 113 p. 119-127. 1997.

$\mathrm{Ma}, \mathrm{W}$. et al. Application of membrane technology for drinking water. Desalination 119. p. 127-131. 1998.

MIERZWA, J.C. Processos de separação por membranas para tratamento de água. Capítulo 8. Contribuição ao estudo da remoção de cianobactérias e microcontaminantes orgânicos por meio de técnicas de tratamento de água para consumo humano. Valter Lúcio de Pádua (coordenador). Rio de Janeiro: ABES, 504 p. 2006.

MIERZWA, J.C.; HESPANHOL, I. Água na Induistria - Uso racional e reúso. Editora Oficina de Textos. São Paulo, 144 p. 2005.

NICOLAISEN, B. Developments in membrane technology for water treatment. Desalination 153 p. 355-360. 2002

SHULZ, C.R.; OKUN, D.A. Surface Water Treatment for Communities in Developing Countries. John Wiley \& Sons. New York, 1984

WILBERT, M.C. et al. The desalting and water treatment manual: A guide to membranes for municipal water treatment. R-98-05. $2^{\text {nd }}$ edition. U.S. Department of the Interior. Bureau of Reclamation. July 1998.

YOON, Y.et al. Nanofiltration and ultrafiltration of endocrine disrupting compounds, pharmaceuticals and personal care products. Journal of Membrane Science. 270, 88-100 p. 2006.

Endereço para correspondência:

José Carlos Mierzwa

Departamento de Engenharia

Hidráulica e Sanitária da EPUSP

Av. Prof. Almeida Prado, 83

Travessa 2 - Prédio da Eng. Civil.

Cidade Universitária

05508-070 São Paulo - SP - Brasil

Tel.: (0I I) 309I-5329

E-mail: mierzwa@usp.br 